\title{
The gut microbiota and graft-versus-host disease
}

\author{
David N. Fredricks \\ Vaccine and Infectious Disease Division and Clinical Research Division, Fred Hutchinson Cancer Research Center, Seattle, Washington, USA. Division of Allergy and Infectious Diseases and Department of \\ Microbiology, University of Washington, Seattle, Washington, USA.

\begin{abstract}
Graft-versus-host disease (GvHD) is a common complication of hematopoietic cell transplantation that negatively impacts quality of life in recipients and can be fatal. Animal experiments and human studies provide compelling evidence that the gut microbiota is associated with risk of GvHD, but the nature of this relationship remains unclear. If the gut microbiota is a driver of GvHD pathogenesis, then manipulation of the gut microbiota offers one promising avenue for preventing or treating this common condition, and antibiotic stewardship efforts in transplantation may help preserve the indigenous microbiota and modulate immune responses to benefit the host.
\end{abstract}

Hematopoietic cell transplantation (HCT) is a life-saving procedure for treating hematological malignancies such as leukemia and lymphoma, and for restoring marrow function in patients with conditions associated with dysfunctional hematopoiesis, such as aplastic anemia. More than 23,000 hematopoietic cell transplants are performed each year in the United States (1), offering hope of cure for patients who have failed to respond to other interventions such as conventional chemotherapy for cancer. The HCT procedure uses preparative regimens consisting of chemotherapy, radiation, radioimmunotherapy, antibody-based immunotherapy, or combinations thereof to deplete native bone marrow cells, immune cells, and residual malignant cells in patients with cancer, which establishes a competitive advantage for donor hematopoietic cells (Figure 1A). The patient then receives an infusion of donor hematopoietic cells, including stem cells, to restore the depleted marrow and rescue hematopoietic function for the production of leukocytes, erythrocytes, and platelets. The source of the donor hematopoietic cells used for transplant can be bone marrow, peripheral blood enriched for stem cells, or banked umbilical cord blood.

Autologous HCT occurs when hematopoietic stem cells are harvested from a cancer patient and reinfused after cytoreductive conditioning (i.e., the patient serves as his or her own donor). This strategy is unsuccessful for conditions such as leukemia, where the reinfused cells may be contaminated by malignant cells. Allogeneic HCT entails collection of hematopoietic stem cells from a (healthy) genetically distinct donor, and the patient is the recipient of the infused cells. The advantages of the allogeneic approach are that malignant cells are not reinfused with the graft, and the donor immune cells can impart an immunologic graft-versus-tumor (GvT) effect wherein they attack and destroy residual host malignant cells, thus helping to effect a cure.

Conflict of interest: The author has declared that no conflict of interest exists. Copyright: @ 2019, American Society for Clinical Investigation.

Reference information: / Clin Invest. 2019;129(5):1808-1817.

https://doi.org/10.1172/JCI125797.

\section{Graft-versus-host disease}

Unfortunately, the ugly flip side of the GvT effect in allogeneic HCT is graft-versus-host disease (GvHD), in which the grafted immune cells recognize normal host tissues as foreign (2). GvHD remains an important cause of death after allogeneic HCT, causing morbidity in $30 \%$ to $70 \%$ of allogeneic HCT recipients (3-5). Even when GvHD does not contribute to mortality, it can have a pernicious effect on quality of life, with some patients debilitated by diarrhea, abdominal pain, nausea, vomiting, wasting, oral ulcers, sclerosis of the skin, and other noxious manifestations $(6,7)$. GvHD is characterized by damage to host tissues and organs mediated by the donor immune cells, particularly donor $\mathrm{T}$ cells that recognize host major and minor histocompatibility alloantigens presented by antigen-presenting cells (8-10). Tissues affected by GvHD may include those in the gastrointestinal tract and oral mucosa, liver, skin, thymus, eye, reproductive tract, and lung.

In acute GvHD, a cascade of $\mathrm{T}$ cell activation, proliferation, and cytotoxic activity propagates the observed tissue damage. HCT conditioning regimens may drive this cascade by causing tissue damage that activates antigen-presenting cells. Acute GvHD is characterized by inflammation that may include dermatitis, enteritis, or hepatic dysfunction and usually develops within 100 days after HCT. Typical manifestations include a maculopapular rash (skin); nausea, vomiting and diarrhea (gut); and rising bilirubin (liver). Chronic GvHD is characterized by oral and ocular sicca, fibrotic complications affecting a wide spectrum of organs, gastrointestinal lesions, or rising bilirubin, usually with onset beyond 100 days yet within 1 to 2 years after transplant $(11,12)$. There is debate regarding whether chronic GvHD is an extension and evolution of acute GvHD or subject to different triggers. Clinical manifestations, rather than onset relative to HCT, determine diagnosis with acute versus chronic GvHD (13). The diagnosis of GvHD is frequently based on clinical findings, such as diarrhea after HCT without another known cause, but tissue biopsy can help support the diagnosis, such as detection of apoptotic cells in the intestinal epithelium and destruction of crypts (14). Several GvHD biomarkers have been studied, but their use is limited in clinical practice (15). Stages of 


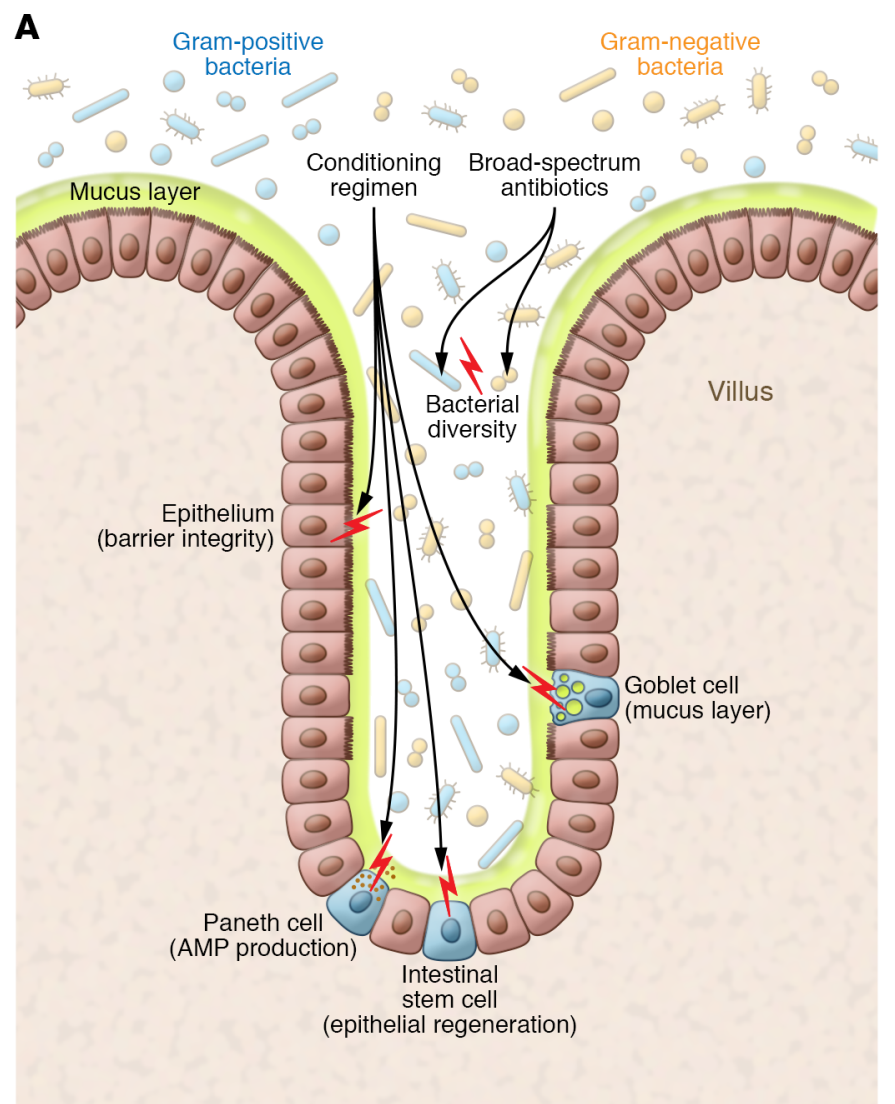

C

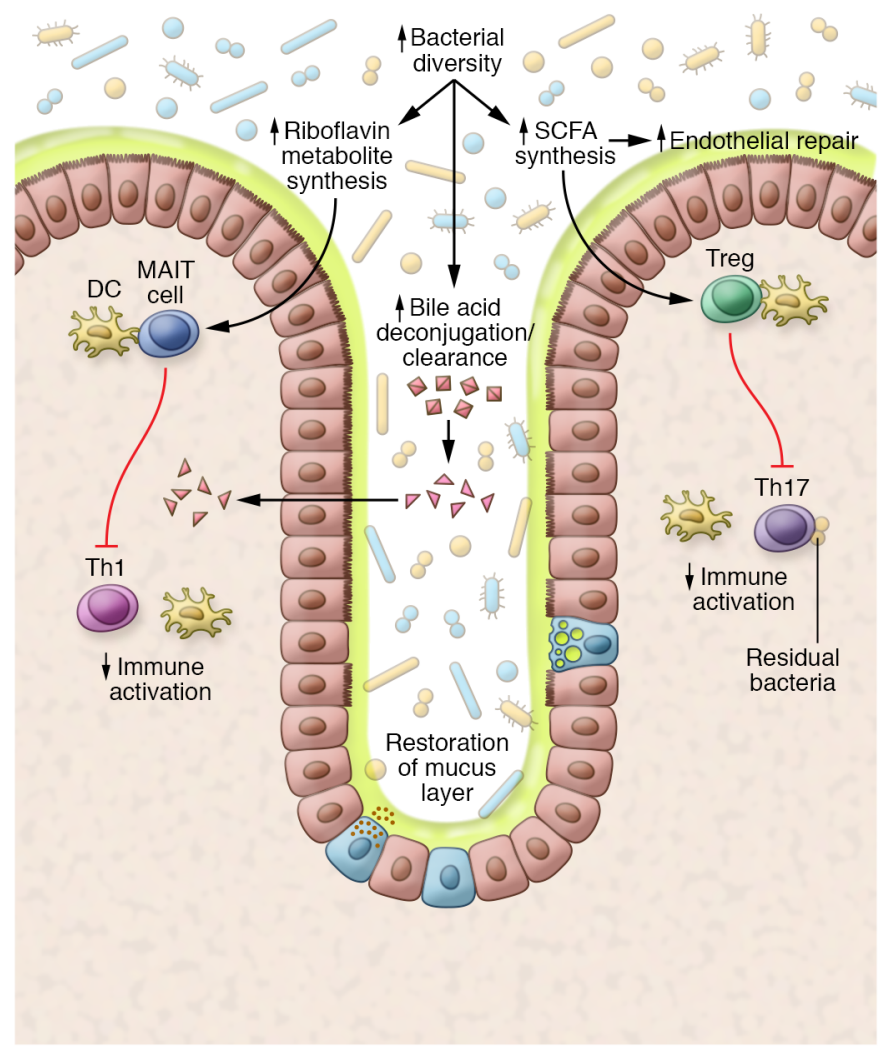

B

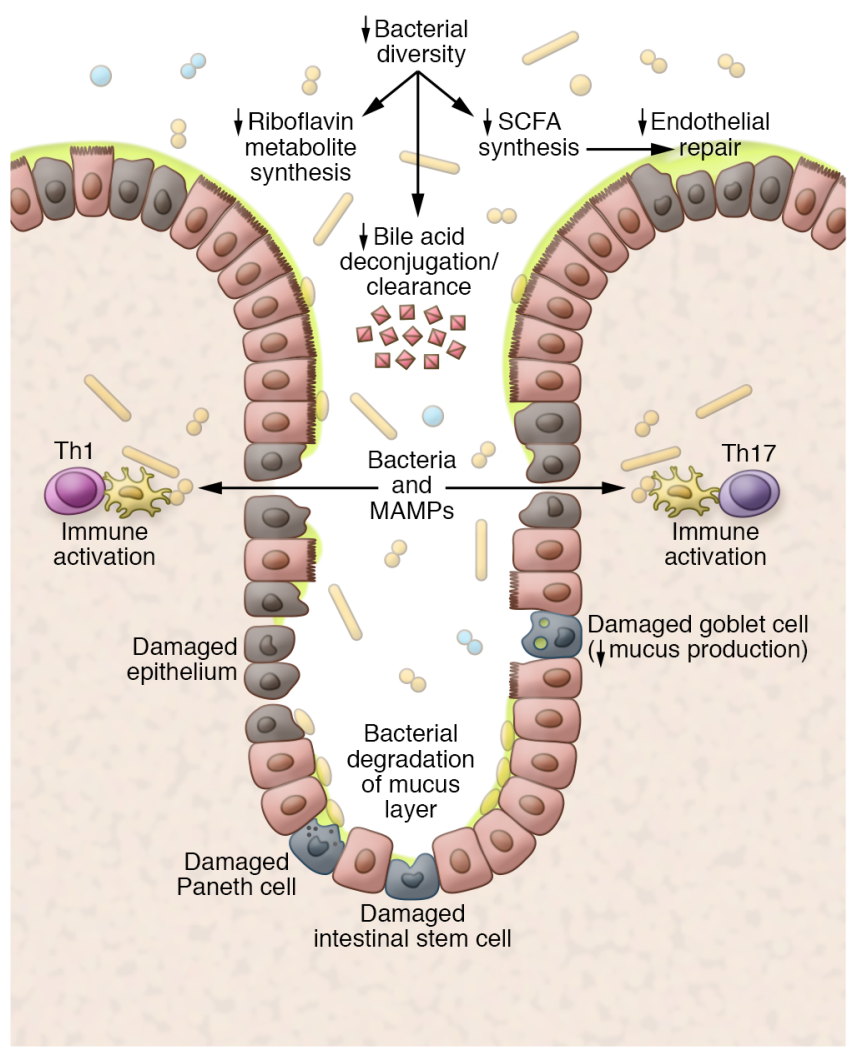

Figure 1. Models of intestinal environments affecting GvHD development. (A) Effects of conditioning. Conditioning chemotherapy and radiation damage cells in the intestinal epithelium (including IECs, thereby compromising barrier function), intestinal stem cells (impairing epithelial regeneration), Paneth cells (decreasing the antimicrobial peptide [AMPs] production that maintains intestinal bacterial populations), and goblet cells (depleting the mucus barrier separating bacteria from epithelium and immune cells). Coincident with conditioning, patients undergoing HCT typically receive systemic broad-spectrum antibiotics intended to prevent bacterial infections during neutropenia; these also disrupt gut microbiota and reduce bacterial diversity. (B) GvHD pathogenesis. Intestinal dysbiosis arising from antibiotics, altered diet, and tissue damage in HCT recipients may deplete riboflavin metabolites and SCFAs (e.g., butyrate), impairing antiinflammatory MAIT cell activation and Treg stimulation, respectively. Depletion of butyrate, the primary energy source for repair-promoting IECs, may also contribute to epithelial defects, allowing ingress of proinflammatory bacteria and MAMPs. Pattern recognition receptors on immune cells like DCs recognize bacteria and MAMPs, eliciting Th1 and Th17 responses that enhance tissue damage. Reduced bacterial diversity may correlate with reduced bile acid metabolism and increased intestinal bile acids, affecting cell function and viability. Overgrowth of mucin-degrading bacteria (e.g., Akkermansia) in the absence of complex dietary carbohydrates may further deplete the mucus barrier, permitting direct bacterial contact with the epithelial surface, activating immune cells. The net effect constitutes a disruption in the gut barrier with inflammation that may trigger and sustain GVHD. (C) Absence of GVHD. Preservation of the gut microbiota, including bacteria with key metabolic properties (riboflavin metabolism, SCFA production), dampens inflammation via MAIT cells and Tregs. Bacterial butyrate production, normal bile acid metabolism and the absence of mucin-degrading bacteria facilitate healing of the gut epithelium. An intact barrier discourages bacterial translocation and MAMPs in the mucosa, resulting in reduced inflammation and reduced alloantigen presentation to $\mathrm{T}$ cells. 
GvHD in each organ (skin, gut, and liver) help to determine the overall peak grade of GvHD in the patient, ranging from grade 0 (no GvHD) to 4 (extreme GvHD) (16).

\section{Prevention and treatment of GvHD}

Approaches to minimize GvHD include optimization of HLA matching between donor and recipient, in vivo or in vitro $\mathrm{T}$ cell depletion, and use of immunosuppressive prophylaxis to inhibit $\mathrm{T}$ cell replication and IL-2 production after HCT (e.g., cyclosporine, tacrolimus, methotrexate, mycophenolate mofetil) (17, 18). Yet, despite such efforts, GvHD remains a major problem after HCT (3-5). Glucocorticoids are standard first-line therapy for acute and chronic GvHD. However, some patients fail to respond, or develop glucocorticoid-resistant disease that portends a grave prognosis. Unfortunately, glucocorticoid treatment can also cause numerous complications. There are strong correlations between initial treatment with high-dose glucocorticoids and increased risks of cytomegalovirus disease and fungal infections $(19,20)$. Noninfectious complications of steroid treatment include osteoporosis, myopathy, avascular necrosis of the hip, and cataract formation.

Responses to second-line immunosuppressive therapies are also poor (21). Secondary treatment trials for GvHD have a long history of failure, and few proven new treatments exist for this condition. Despite decades of research, rates of chronic GvHD continue to rise (3). Curiously, some HCT recipients with excellent HLA matching to their HCT donor still develop GvHD, while other recipients with less optimal HLA matching do not develop GvHD. Furthermore, some patients undergoing autologous HCT develop a syndrome of pseudoGvHD that responds to steroids, which does not fit current models of pathophysiology since no mismatch exists in either major or minor tissue antigens of graft and recipient tissue. Clearly, our understanding of GvHD drivers is incomplete. New paradigms are needed to better understand GvHD pathogenesis, because opportunities to develop new approaches for prevention and treatment will arise based on this knowledge. As our patients continue to die with treatment-refractory GvHD, it is time to think more broadly about this disease.

\section{The microbiota and GvHD}

Human mucosal surfaces and skin harbor a complex microbiota of bacteria, archaea, fungi, other protists, and viruses, with each body site (or niche) colonized with a distinct consortium of microbes. These consortia are referred to as the microbiota, whereas the microbiome refers to the microbiota, their associated genes, and their environment. The microbiota is critical for maintaining healthy tissues and stimulating immunity, but unhealthy alterations in microbial populations (dysbiosis) have been linked to disease and defects in immunity. GvHD most commonly affects tissues that have a microbiota, such as the gut, mouth, and skin, or the liver (which is seeded with microbial products from the portal circulation). What is the evidence that the microbiota may influence GvHD? Surprisingly, in the 1970s some of the earliest animal studies of bone marrow transplantation hinted at a role for the microbiota in GvHD, and this evidence continues to accumulate. For a detailed bibliography on the microbiota and HCT outcomes, please see several informative recent reviews (22-24). This Review will focus on the microbiota as it relates to GvHD, and specifically the bacterial biota, though fungi and viruses may also play a role.

\section{Animal models}

In 1965, Connell and Wilson showed that germ-free mice undergoing bone marrow transplantation had prolonged survival compared with conventional mice, but the mechanism for this benefit was not evident (25). In 1971 Jones et al. demonstrated that germ-free mice receiving an allogeneic bone marrow transplant were protected from GvHD (then called secondary disease) and had prolonged survival compared with mice with a conventional microbiota (26). Conventionalizing these germ-free mice (i.e., colonizing with microbes) after transplantation induced GvHD, resulting in death. In 1974 van Bekkum et al. confirmed that germ-free mice were protected from GvHD in their transplant model, but they did not see consistent onset of GvHD following conventionalization (27). Protection from GvHD in germ-free animals has been confirmed in other studies (28), including with cross-species (xenogeneic) transplants that typically elicit more severe GvHD due to alloantigen mismatch $(29,30)$. There are several possible interpretations of this research. In one model, the microbiota plays a direct role in promoting tissue injury or inflammation after HCT, and therefore absence of a microbiota in germ-free animals protects against GvHD by eliminating this inciting factor. However, animals raised in germ-free conditions have abnormal development, with highly aberrant immunity (31-33). Therefore, an alternative explanation for these results posits that the process of raising animals under germ-free conditions produces an immunologically deranged host that is less capable of developing GvHD. In the first model, microbes are direct actors, while in the second model, microbes indirectly shape the immune response that influences GvHD. In actuality, both direct and indirect effects may occur simultaneously.

Compelling evidence for a direct role of the microbiota in GvHD would come from animal models with normal immunological development but an altered microbiota. van Bekkum and colleagues were the first to demonstrate protection from GvHD in such a model: mice subjected to gut decontamination with antibiotics were protected from GvHD and death after transplant (27). Subsequent studies using antibiotic-mediated gut decontamination in mice (34) and dogs (35) corroborated these findings, supporting the notion that microbes - not just deranged immune development - most proximately affect the development of GvHD. Unfortunately, few animal studies have measured the impact of antibiotic-mediated gut decontamination on the resulting gut microbiota, or linked particular bacterial species to GvHD. However, Jenq and colleagues focused on the association between intestinal bacterial composition and GvHD in a mouse model. Using advanced molecular methods (36), they demonstrated that depletion of Lactobacillus species (using ampicillin) was associated with worse GvHD, while replacement of lactobacilli was associated with protection. These investigators proposed that the presence of lactobacilli such as L. johnsonii in the gut helped to protect from domination with Enterococcus species and Enterobacteriaceae, which are linked to GvHD. These data emphasize that not all bacteria have the same effect on GvHD: the collection of bacterial species and their function matter. Simms-Waldrip et al. analyzed the stool microbiota of pediatric patients undergoing HCT and noted that loss of key Clostridia such as Clostridium leptum and Eubacterium rectale was associated with increased GvHD; these results were replicated in a mouse model of GvHD (37). These animal studies 
provide convincing evidence that microbes have a role in modulating GvHD in well-controlled experimental systems.

\section{Human studies}

It is not feasible to make humans "germ-free" to assess the impact of the microbiota on GvHD risk, but it is possible to alter the microbiota using antibiotics, in either observational studies or controlled trials, and assess the effect on GvHD. Several studies performed at the Fred Hutchinson Cancer Research Center examined the impact of oral antibiotics combined with protective isolation or laminar airflow rooms as an intervention against infectious complications and GvHD compared with standard care. Use of oral antibiotics for gut decontamination reduced incidence of acute GvHD or delayed GvHD onset in some studies and patient populations $(38-40)$, but lacked benefit in other studies $(41,42)$. Heterogeneity in study design and interventions may explain this lack of consistency. Vossen et al. demonstrated lower rates of acute and chronic GvHD in pediatric patients undergoing allogeneic HCT when treated with nonabsorbable antibiotics for complete gut decontamination, compared with patients receiving antibiotics for selective gut decontamination (43). In a follow-up study using a variety of different antibiotics, Vossen and colleagues demonstrated that successful gut decontamination in pediatric HCT recipients reduced incidence of acute GvHD, compared with those without successful decontamination (44). These studies raise several questions: are differences in efficacy in these studies related to differences in the antibiotics used for gut decontamination, antibiotic efficacy, hosts (e.g., study population, underlying disease, conditioning regimen), GvHD prophylaxis, power of each study to detect a difference, or other factors? One critical factor missing from these studies is detailed analysis of the microbiology.

In 1992, Beelen et al. showed that allogeneic HCT recipients experienced reduced rates of GvHD when treated with oral, nonabsorbable antibiotics if they had sustained depletion of anaerobic bacteria as measured by stool culture (45). This observation formed the basis for a randomized, open-label trial of anti-anaerobic antibiotic therapy (metronidazole) to deplete gut anaerobes linked to GvHD (46). Oral metronidazole plus oral ciprofloxacin was compared with oral ciprofloxacin alone for prophylaxis and gut decontamination in adult patients undergoing allogeneic HCT (46), with the expectation that metronidazole would be effective in depleting gut anaerobes whereas ciprofloxacin would not. Among the 134 study participants, the incidence of grades II-IV acute GvHD was $52 \%$ in the ciprofloxacin arm and $25 \%$ in the metronidazole plus ciprofloxacin arm. This striking halving of acute GvHD incidence was associated with reduced anaerobic bacterial colony counts in stool. The effect of metronidazole on acute GvHD was most evident for recipients from HLA-matched sibling donors, and there was no significant impact on chronic GvHD or overall survival. Stephen Guthery and colleagues performed an open-label pilot study of oral metronidazole prophylaxis in pediatric patients undergoing allogeneic HCT, assessing GvHD incidence in comparison with historical controls (47). In a multivariate analysis, the relative risk of grades II-IV GvHD was $0.36(P=0.05)$ for the 19 evaluable patients receiving metronidazole, providing evidence of protection from GvHD. None of these studies characterized the stool microbiota using advanced molecular methods. Despite evidence in both adult and pediatric HCT recipients that metronidazole use is associated with reduction in acute GvHD, no additional trials have been published, and metronidazole has not been adopted as a standard approach to reduce GvHD incidence or severity.

More recently, seminal studies have used advanced molecular microbiological methods to further elucidate the link between the human microbiota and GvHD. Robert Jenq, Carles Ubeda, and colleagues studied changes in the gut microbiota of humans undergoing allogeneic HCT, including eight patients with GvHD and ten controls without GvHD (36). Patients experiencing GvHD had decreased stool microbial diversity, increased lactobacilli abundance, and decreased representation of bacteria in the Clostridiales order. Allogeneic HCT was associated with major shifts in the gut microbiota, with severe perturbations associated with GvHD risk. Another study by Ying Taur and colleagues showed that loss of bacterial community diversity in stool at time of engraftment post-HCT was associated with reduced overall survival and increased transplant-related mortality (48). For example, survival at 3 years after HCT was $36 \%, 60 \%$, and $67 \%$ for groups with low-, intermediate-, and high-diversity gut microbiota, respectively $(P=$ 0.019). These findings generated enthusiasm for efforts to restore gut bacterial diversity after HCT. The challenge in interpreting this study is common to the microbiome field: we do not know whether low gut microbial diversity is a driver of mortality or a marker of another process linked to mortality. For example, it is possible that a low-diversity microbiota drives mortality through loss of protective bacteria that normally promote healthy gut barrier function and immune tolerance, and/or through gain of dominant pathobiont populations that produce inflammation, infection (e.g., bacteremia), and GvHD. Alternatively, rather than being in a causal pathway for mortality, low gut microbial diversity may simply be a marker of a disturbed microbial ecosystem. Low microbial diversity could, for example, indicate receipt of previous courses of antibiotics, which are linked to prior courses of chemotherapy, which in turn are linked to uncontrolled malignancy and tissue toxicity. An interventional study designed to restore bacterial diversity would be the only definitive way to prove that gut microbial diversity is a driver of mortality. In contrast to its association with mortality, no demonstrable association was observed between stool bacterial diversity and GvHD itself in the study by Taur and colleagues. However, as noted by Jenq et al. in 2015 (49), Taur's study likely lacked the power to fully probe this relationship because patients received T cell-depleted grafts that reduce GvHD incidence.

To overcome this limitation, Jenq et al. investigated the connection between the gut microbiota and GvHD in 64 HCT recipients of T cell-replete grafts, revealing an association of Blautia bacterial species with reduced risk of GvHD-related mortality, reduced use of steroids for treatment of GvHD, and increased overall survival. This association demonstrated excellent reproducibility in a validation study of $51 \mathrm{HCT}$ recipients of T cell-replete grafts at the same institution (49). Interestingly, Blautia species were not associated with clinical diagnosis of GvHD, but rather GvHD-related mortality. Low gut bacterial diversity was also associated with increased GvHD-related mortality, consistent with previous studies. Importantly, the association between Blautia species and GvHD means that key microbial taxa may mitigate inflammation in GvHD, and could be used in a probiotic approach to prevent GvHD if it behaves 
as a driver in this process. For example, evidence in a murine model links use of the probiotic strain of Lactobacillus rhamnosus GG with reduced GvHD and improved survival (50). But mice are not humans, and we need to identify bacteria associated with reduced GvHD risk in humans. Indeed, a randomized controlled trial of L. rhamnosus GG probiotic in human allogeneic HCT recipients demonstrated no benefit in preventing GvHD (51).

In a study of 31 patients undergoing allogeneic HCT, Holler, Butzhammer, and colleagues examined the stool microbiota using broad-range 16S rRNA gene PCR with pyrosequencing and taxonspecific endpoint PCR for Enterococcus species (52). Patients received prophylactic antibiotics consisting of ciprofloxacin and metronidazole from day 0 of transplant until engraftment, in addition to pretransplant trimethoprim-sulfamethoxazole, and other antibiotics for infections. PCR studies demonstrated loss of bacteria in the Firmicutes phylum, and an association of GvHD risk with both relative abundance and prevalence of enterococci. The authors also measured urinary concentrations of indoxyl sulfate, a metabolite of tryptophan that is produced by commensal gut bacteria and excreted in urine. Low levels of urinary indoxyl sulfate were associated with antibiotic use and GvHD, and levels nadired at the time of neutropenia. Urinary indoxyl sulfate is a potential biomarker of gut bacterial community disruption and GvHD risk, as well as other negative outcomes (53).

Biagi et al. examined the gut microbiota in ten pediatric patients undergoing HCT, noting lower relative abundance of Bacteroides and Parabacteroides species in patients who went on to develop acute GvHD. Furthermore, they showed lower levels of propionate (a short-chain fatty acid [SCFA]) in stool samples from subjects with GvHD (54). Doki et al. examined the stool microbiota of 107 allogeneic HCT recipients prior to transplant and noted little ability to predict GvHD, and no association between stool bacterial diversity and overall survival, relapse, nonrelapse mortality, or GvHD (55). This study suggests that the gut microbiota may be more informative in predicting GvHD after transplant, such as at engraftment, than before transplant, as noted below.

Golob et al. analyzed the gut microbiota from stool samples collected weekly in 66 patients undergoing allogeneic HCT (56). Using broad-range 16S rRNA gene PCR with next-generation sequencing, they determined that severe acute GvHD of the gut was associated with reduced bacterial alpha diversity (i.e., site-specific species diversity and evenness) in stool at engraftment, confirming a finding noted by other investigators (49). The decline in bacterial diversity in stool is expected, given multiple antibiotics administered to HCT recipients for prophylaxis, empiric therapy, or directed therapy, but the degree of change was linked to GvHD. Absence of members of the Lachnospiraceae family (including particular Blautia species) at the time of neutrophil engraftment was associated with increased risk of GvHD, while the presence of oral Actinobacteria and oral Firmicutes in the gut was associated with increased risk of GvHD. A metric was calculated to predict GvHD risk based on the sum of relative abundances of bacteria positively associated with GvHD risk minus the sum of relative abundances of bacteria negatively associated with GvHD risk. A receiver operating characteristic curve for this gradient had an area under the curve of 0.83 , suggesting that assessment of stool microbiota at the time of engraftment has modest ability to predict GvHD onset. This analysis significant- ly associated numerous gut bacterial species from different phyla with decreased or increased risk of developing GvHD. In contrast, a study by C. Liu and colleagues (56) did not show an association between acute GvHD and stool bacterial taxa or diversity in the 57 recipients analyzed, but they did note that higher bacterial diversity in the donor was associated with reduced risk of GvHD in the recipient in 22 studied pairs. The mechanism for this association is not clear. A retrospective study by Routy et al. (57) found that allogeneic HCT recipients receiving mostly quinolone antibiotic prophylaxis had higher rates of GvHD compared with patients not receiving antibiotic prophylaxis, though multiple differences between the compared patient groups, including conditioning regimens and cell source, may have influenced these results.

Common findings from these important studies link the gut microbiota to GvHD. However, differences in antibiotic-use practices, individual antibiotic histories, hosts (underlying cancer or condition, previous chemotherapy), treatment protocols (including conditioning regimens, $\mathrm{T}$ cell depletion of grafts), and other unmeasured factors across transplant centers can lead to heterogeneity in findings. If these scientific findings are to be broadly useful to the larger population of patients undergoing allogeneic HCT, then we need to identify common patterns and mechanisms linking the microbiota to GvHD risk. For example, almost all studies associate reduced stool bacterial diversity with GvHD risk or mortality. Enterococci have been associated with elevated risk of GvHD in several studies, but the role of lactobacilli in GvHD is less clear $(36,52,56)$. It is possible that lactobacilli increase in concentration before onset of GvHD, and yet still offer some protection in comparison with subjects without lactobacilli. Results derived from a mouse model by Jenq and colleagues (36) are consistent with this scenario, highlighting that while microbes may be markers of GvHD risk, modulators of GvHD risk, or both, the directionality of these associations can be complex.

\section{The microbiota paradox in GvHD}

Animal and human studies noted above illuminate a possible conceptual conflict. Evidence of protection from GvHD exists in germfree animals, animals treated with antibiotics for gut decontamination, and humans treated with oral nonabsorbable antibiotics or metronidazole (targeting anaerobes) for gut decontamination, suggesting that gut bacteria promote development of GvHD. Yet human and animal studies also demonstrate that protection from GvHD is associated with high alpha diversity and high abundance of anaerobes in the Clostridia class and Lachnospiraceae family, suggesting a beneficial effect of gut microbes against GvHD. How can these discordant observations be true? It is possible that complete absence of bacteria in the gut removes microbial products as triggers of inflammation and this effect dominates in protecting from GvHD. On the other hand, if complete gut decontamination is not achieved, then the remaining bacterial community may shape GvHD risk in different directions, with some bacteria, such as Enterococcus species, increasing risk and other bacteria, such as Blautia species, decreasing risk. The degree of GvHD risk may thus reflect the balance of beneficial and deleterious bacteria present in the gut. For example, it is possible that metronidazole use allows for growth of key Lachnospiraceae associated with health while depleting some Bacteroides species associated with disease. 
Table 1. Mechanisms by which gut microbiota may influence GvHD

\begin{tabular}{|c|c|c|c|}
\hline Mechanism & Role of microbiota & Host involvement & References \\
\hline $\begin{array}{l}\text { Bacterial translocation- } \\
\text { associated inflammation }\end{array}$ & $\begin{array}{c}\text { Bacteria and associated MAMPs translocate the disrupted } \\
\text { gastrointestinal barrier }\end{array}$ & $\begin{array}{l}\text { Pattern recognition receptors recognize bacterial MAMPs, } \\
\text { recruit inflammatory cells }\end{array}$ & $50,60,61$ \\
\hline $\begin{array}{l}\text { Impaired intestinal } \\
\text { barrier integrity }\end{array}$ & $\begin{array}{l}\text { Dysbiosis (reduced Clostridiales abundance) reduces production } \\
\text { of SCFAs including butyrate, a primary energy source for IECs that } \\
\text { induces Tregs and inhibits HDAC }\end{array}$ & $\begin{array}{l}\text { Limited butyrate hinders IEC recovery and wound healing, } \\
\text { decreasing barrier integrity }\end{array}$ & $63,64,66$ \\
\hline $\begin{array}{l}\text { Altered balance of Tregs } \\
\text { versus Th1/Th17 cells }\end{array}$ & & $\begin{array}{l}\text { Lower butyrate levels limit homing of Tregs to gut mucosa, } \\
\text { alter signaling through inflammatory pathways }\end{array}$ & $70,91,92$ \\
\hline $\begin{array}{l}\text { Reduced antimicrobial } \\
\text { peptide (AMP) production }\end{array}$ & $\begin{array}{l}\text { Maintains gut microbiota diversity and balance; increasing } \\
\text { AMP ( } \alpha \text {-defensin) production prevents GvHD-mediated } \\
\text { dysbiosis in mice }\end{array}$ & $\begin{array}{l}\text { Loss of Paneth cells, which produce AMPs in the small intestine, } \\
\text { is a marker of GvHD risk. Production of AMPs ( } \alpha \text {-defensins) } \\
\text { by Paneth cells prevents GvHD-mediated dysbiosis. }\end{array}$ & 71-74 \\
\hline Loss of mucus barrier & $\begin{array}{l}\text { May increase opportunities for bacteria and MAMPs to stimulate } \\
\text { immune cells }\end{array}$ & $\begin{array}{l}\text { Loss of goblet cells, which produce mucus, has been linked to GvHD. } \\
\text { Imipenem-cilastatin treatment in mice with GvHD is associated with } \\
\text { increased levels of the mucinolytic bacterium Akkermansia muciniphila, } \\
\text { thinning of the mucus layer, and compromised intestinal barrier function. }\end{array}$ & 75,93 \\
\hline IL-17 & $\begin{array}{l}\text { Transfer of intestinal microbiota from IL-17 receptor-KO mice } \\
\text { confers increased susceptibility to acute GvHD in WT mice }\end{array}$ & IL-17A derived from HCT recipient mice is protective against acute GvHD & 95 \\
\hline MAIT cells & $\begin{array}{l}\text { Activated by riboflavin metabolites produced by some gut bacteria; } \\
\text { influence gut microbiota composition in mice; reconstitution is } \\
\text { associated with increased Blautia and Bifidobacterium longum } \\
\text { abundance in the gut in humans }\end{array}$ & $\begin{array}{l}\text { HCT recipient MAIT cells protect from GvHD in mice, constrain alloreactive } \\
\text { donor T cell expansion in colon, and produce IL-17A. In humans, robust } \\
\text { MAIT cell reconstitution is associated with reduced risk of subsequent } \\
\text { acute GvHD; MAIT cells suppress proliferation of conventional T cells. }\end{array}$ & 96,97 \\
\hline Tryptophan & Lactobacilli produce tryptophan metabolites that activate ILCs & Activated ILCs produce IL-22 (see IL-22 below) & 98 \\
\hline IL-22 & Shapes the gut microbiota of mice & $\begin{array}{l}\text { Promotes intestinal stem cell-mediated epithelial regeneration } \\
\text { and is associated with reduced CvHD severity in mice }\end{array}$ & $99-101$ \\
\hline
\end{tabular}

HDAC, histone deacetylase; IEC, intestinal epithelial cell; ILC, innate lymphoid cell; MAIT cell, mucosal-associated invariant T cell; MAMP, microbeassociated molecular pattern; SCFA, short-chain fatty acid.

If this model is true, two potential strategies emerge for reducing GvHD in humans. Eradicating all bacteria from the gut and maintaining this state is likely to be a difficult task indeed, owing to challenges with bacterial antibiotic resistance and exposure to nonsterile environments even when patients are in protected isolation. A more feasible alternative might be to engineer the gut microbiota of HCT recipients, augmenting with strains of bacteria that outcompete pathobionts such as Enterococcus and the Enterobacteriaceae, while providing critical microbial metabolites such as butyrate, which facilitates regrowth of intestinal epithelial cells and dampens inflammation by promoting regulatory $\mathrm{T}$ cell (Treg) activity. All patients will eventually be "conventionalized" with gut microbiota after cessation of antibiotics. Therefore, efforts should be focused on preserving the diversity of HCT recipients' gut microbiota, including bacteria associated with optimized gut function and immunity, while supplementing patients with beneficial bacteria when they have evidence of diversity loss, mono-domination by pathobionts, and dysbiosis. These efforts may include optimizing antibiotic selection to preserve gut bacterial diversity, using oral $\beta$-lactamase enzymes to degrade intraluminal antibiotics $(58,59)$, administering live organisms that protect from GvHD, and using diet and prebiotics to promote growth of beneficial bacterial taxa.

\section{Mechanism}

Multiple mechanisms have been proposed to explain how the gut microbiota influence the development of GvHD (Table 1). The microbiota may stimulate host cell inflammation via influx of bacteria or microbe-associated molecular patterns (MAMPs) across an epithelial barrier disrupted by conditioning chemotherapy and radiation $(50,60,61)$. These bacteria and MAMPs may then interact with pattern recognition receptors on human cells, recruiting inflammatory cells operative in GvHD. The multiple immune cells and pathways linked to GvHD pathogenesis in animal and human studies are summarized in Table 1.

In addition, the gut microbiota can influence host cell physiology via production of metabolites such as the SCFA butyrate (e.g., by Lachnospiraceae) (62). Butyrate is a primary energy source for intestinal epithelial cells, but also impacts inflammatory signaling pathways (63) and improves wound healing in the intestine at high concentrations (64). In a murine model, SCFAs made by the gut microbiota attenuated GvHD (65), and low butyrate levels in intestinal tissue were associated with GvHD (66). Furthermore, gavage with butyrate or a Clostridia mixture reduced GvHD independently of Treg number (66). Butyrate also functions as a histone deacetylase inhibitor to inhibit antigen-stimulated $\mathrm{T}$ cells 


\section{Table 2. Clinical data on microbiota-linked interventions for GvHD}

\begin{tabular}{|c|c|c|c|c|}
\hline Intervention & Total patients & Study design & Outcome & Reference \\
\hline \multirow[t]{3}{*}{$\begin{array}{l}\text { Fecal microbiota } \\
\text { transplant (FMT) }\end{array}$} & 4 & $\begin{array}{l}\text { Related stool donor. Delivered via nasoduodenal tube to patients } \\
\text { with acute, steroid-refractory or -dependent GvHD. }\end{array}$ & Complete resolution in 3 of 4 patients (partial in 1) & 102 \\
\hline & 3 & $\begin{array}{l}\text { Related or anonymous stool donor. Delivered via colonoscopy } \\
\text { to patients with acute, refractory GvHD. }\end{array}$ & Complete resolution in 2 of 3 patients (partial in 1) & 103 \\
\hline & 13 & $\begin{array}{c}\text { Healthy, third-party stool donors. Delivered by oral capsule } \\
\text { to patients after HCT. }\end{array}$ & $\begin{array}{l}\text { Improvement of intestinal microbiome diversity after FMT, } \\
\text { associated with expansion of stool-donor taxa }\end{array}$ & 85 \\
\hline \multirow[t]{2}{*}{ Probiotics } & 30 & Lactobacillus plantarum. Single-arm study in pediatric patients. & $\begin{array}{l}\text { No Lactobacillus bacteremia reported; } 97 \% \text { of children received } \\
\qquad \geq 50 \% \text { of doses }\end{array}$ & 105 \\
\hline & 31 & $\begin{array}{l}\text { Lactobacillus rhamnosus GG. Randomized, controlled study in adults. } \\
\qquad n=20 \text { for probiotic group; } n=13 \text { for control. }\end{array}$ & No difference in gut microbiota diversity or GvHD incidence & 51 \\
\hline & 394 & $\begin{array}{l}\text { Retrospective analysis of allogeneic HCT patients receiving rifaximin } \\
\text { vs. ciprofloxacin/metronidazole. } n=194 \text { for rifaximin group; } \\
\qquad n=200 \text { for cipro/metro group. }\end{array}$ & $\begin{array}{l}\text { Patients on rifaximin had lower enterococcal positivity, higher urinary } \\
\text { 3-indoxyl sulfate concentrations, and lower gastrointestinal } \\
\text { GvHD-related TRM }\end{array}$ & 107 \\
\hline & 621 & $\begin{array}{l}\text { Retrospective analysis of timing of peritransplant antibiotic treatment } \\
\text { in allogeneic HCT recipients. } n=236 \text { for early antibiotic group; } \\
n=297 \text { for late antibiotic group; } n=88 \text { for no antibiotic group. }\end{array}$ & $\begin{array}{l}\text { Early antibiotic exposure was associated with decreased } \\
\text { Clostridiales, lower urinary 3-indoxyl sulfate, and higher } \\
\text { GvHD-associated TRM }\end{array}$ & 84 \\
\hline \multirow[t]{2}{*}{$\beta$-Lactamase } & 24 & $\begin{array}{l}\text { Phase lla trials to confirm that ribaxamase degrades } \beta \text {-lactam } \\
\text { antibiotics in the human intestine. }\end{array}$ & $\begin{array}{l}\text { Ribaxamase degraded ceftriaxone to below the level of quantitation } \\
\text { and was well tolerated in human subjects }\end{array}$ & 59 \\
\hline & 412 & $\begin{array}{l}\text { Phase llb trial to measure ability of ribaxamase to prevent changes in } \\
\text { the gut microbiota and C. difficile cases following ceftriaxone treatment. }\end{array}$ & $\begin{array}{l}\text { Ribaxamase preserved gut microbiota diversity (significant difference } \\
\text { in beta diversity between ribaxamase-treated and control patients) }\end{array}$ & 60 \\
\hline
\end{tabular}

that mediate GvHD (66-70). These data suggest a mechanistic link between gut bacteria, metabolites, and GvHD risk.

Changes to patients' gastrointestinal tracts during HCT may in turn affect the gut microbiota. For example, overgrowth of pathobionts may result from a loss of antimicrobial peptide-producing Paneth cells in the small intestine (71-73). Indeed, loss of Paneth cells is a marker of GvHD risk in humans (74). Similarly, loss of goblet cells and the associated mucus barrier excreted by these cells has been linked to GvHD (75). The large intestine's mucus layer keeps the microbiota distant from epithelial cells, and loss of this layer may increase opportunities for stimulation of immune cells. Loss of Paneth cells in the small intestine, loss of the mucus barrier in the large intestine, and disruption of epithelial barriers due to conditioning chemoradiotherapy may create a perfect storm of microbe-driven inflammation in the gut (Figure 1B).

\section{The future}

Research investigating the microbiota and GvHD has great potential to illuminate the pathophysiology of GvHD, but also shed light on how interventions such as antibiotics and cancer therapy impact the microbiota, and how the microbiota influences immunity, autoimmunity, tissue repair, and physiology (Figure 1C). Patients undergoing allogeneic HCT are a biological extreme; they lack a functioning immune system in the pre-engraftment period, experience a new immune system at engraftment, and are subject to radical changes in their microbiota while receiving antibiotics and recovering from conditioning-related tissue damage. Important findings are likely to emerge at this extreme of biology, but attention to key concepts will help expedite progress:

Descriptive versus mechanistic investigations. We are still in an era of describing how the microbiota is associated with GvHD, and this is appropriate. One needs to know the actors to fully understand the actions of the microbiota. However, future studies examining mechanism have the potential to identify interventions that will help translate these associations into therapies for the treatment and prevention of GvHD if the microbiota is causal. For example, understanding how the gut microbiota metabolizes host-derived compounds to promote or ameliorate inflammation may lead to new drugs that treat GvHD by blocking or augmenting these pathways, respectively.

Elucidating the microbiota-GvHD connection. Although many investigators have established a connection between the microbiota and $\operatorname{GvHD}(24,26,27,34,36,44,49,52,66,71,72,76-82)$, it remains 
unclear which gut bacteria are most linked to GvHD risk in humans, and whether changes in the gut microbiota cause GvHD, result from GvHD, or both. Studies in humans that manipulate the microbiota and then measure GvHD and microbiological outcomes will help advance the field. These interventions may include truncated use of antibiotics in afebrile neutropenic patients (83), or improved antibiotic stewardship with reduced use of antibiotics such as carbapenems and piperacillin-tazobactam to preserve gut microbial diversity (84). Alternatively, active measures should be explored to determine whether fecal microbiota transplants (85), engineered microbial consortia, or nutritional/prebiotic interventions reduce GvHD risk and successfully treat patients with GvHD.

Improving taxonomic resolution. Despite use of modern molecular microbiological methods, such as consensus sequence $16 \mathrm{~S}$ rRNA gene PCR with next-generation sequencing to assess bacterial community composition, there is inadequate taxonomic resolution in some studies. Many 16S rRNA gene analyses only provide classification of bacteria at the genus, family, order, or even phylum level, lumping disparate bacteria into a single designation. Different bacterial species within a genus can have different functional and immunological impacts. For example, our data show that certain Bacteroides species are associated with increased risk of GvHD whereas others are associated with reduced risk (86); lumping these different bacteria into the Bacteroides genus for analysis would miss this differential effect. Even species-level designation may be inadequate in circumstances in which bacterial strains within a species have different capabilities and functions, highlighting the value of true metagenomics studies (sampling all DNA, not just rRNA genes) to assess community gene content and function. It is difficult to achieve consensus in the field when different studies identify the same $16 \mathrm{~S}$ rRNA gene sequence but report different taxonomic designations for the bacterium. Accurate species- and strain-level identification is necessary to move the field forward.

Understanding temporal dynamics. Time is a critical element to study when linking the gut microbiota to GvHD risk. It is imperative to determine whether changes in the gut microbiota precede or follow the development of GvHD, or both. This requires detailed longitudinal studies with frequent sample collection throughout the transplant period, including before transplant, at engraftment, and for the period of GvHD risk. Cross-sectional studies cannot inform regarding the directionality of the association between changes in bacterial populations and gut pathology. Knowing that a population of microbes change before the onset of pathology is useful in determining whether microbes are drivers or passengers in GvHD.
Opportunities for change. The human gut microbiota is relatively stable and diverse, making it difficult to change the microbiota in healthy people using interventions such as probiotics (87). But patients undergoing allogeneic HCT tend to lose bacterial diversity, as with Clostridium difficile colitis $(58,88)$. This situation creates an opportunity to intervene by reestablishing diversity using microbes that modulate inflammation.

Moving beyond probiotics. The disrupted gut microbiota in allogeneic HCT recipients may enable reformulation of the microbiota using interventions such as fecal microbiota transplants and administration of engineered microbial communities that restore complete microbial communities. Studies of fecal microbiota transplantation and other interventions are under way and may provide more direct evidence for the microbiota's role in GvHD (Table 2).

Genetic and environmental influences. The host, microbiota, and environment interact to influence propensity for disease. There are many studies focused on how the microbiota and environment interact to affect GvHD, but we also need to explore how host genetics, and particularly immunogenetics, influence the pathogenesis of GvHD $(77,89)$. Patients undergoing HCT may have increased or decreased inflammatory responses to commensal microbes due to single-nucleotide polymorphisms in innate pattern recognition receptors, which may affect GvHD incidence and severity (76). In addition, commensal microbial production of butyrate may affect host cell proteins such as the epithelial butyrate receptor peroxisome proliferator-activated receptor- $\gamma$, leading to decreases in nitrate and oxygen levels that inhibit the replication of Enterobacteriaceae (90). In the absence of butyrate-producing commensals, disrupted host signaling may promote the emergence of pathobionts. We need to study the host as well as microbes, in defined environments, to truly understand how microbes may contribute to GvHD.

\section{Acknowledgments}

Thanks to Ashley Sherrid for designing the figure and for editorial assistance. Thanks to Marco Mielcarek for helpful comments on the manuscript. This work was funded by NIH R01AI134808.

Address correspondence to: David N. Fredricks, Fred Hutchinson Cancer Research Center, Vaccine and Infectious Disease Division, 1100 Fairview Avenue North, E4-100, Seattle, Washington 98109, USA. Phone: 206.667.1935; Email: dfredric@fredhutch.org.

\footnotetext{
1. D'Souza A, Fretham C. Current uses and outcomes of hematopoietic cell transplantation (HCT): CIBMTR summary slides, 2017. CIBMTR website. https://www.cibmtr.org/ReferenceCenter/ SlidesReports/SummarySlides/pages/index.aspx. Updated June 13, 2018. Accessed March 7, 2019.

2. Ferrara JL, Levine JE, Reddy P, Holler E. Graft-versus-host disease. Lancet. 2009;373(9674):1550-1561.

3. Arai S, et al. Increasing incidence of chronic graftversus-host disease in allogeneic transplantation: a report from the Center for International Blood and Marrow Transplant Research. Biol Blood Marrow Transplant. 2015;21(2):266-274.

4. Jacobsohn DA, Vogelsang GB. Acute graft versus
}

host disease. Orphanet J Rare Dis. 2007;2:35.

5. Storb R, et al. Allogeneic hematopoietic cell transplantation following minimal intensity conditioning: predicting acute graft-versus-host disease and graft-versus-tumor effects. Biol Blood Marrow Transplant. 2013;19(5):792-798.

6. Gratwohl A, et al. Acute graft-versus-host disease: grade and outcome in patients with chronic myelogenous leukemia. Working Party Chronic Leukemia of the European Group for Blood and Marrow Transplantation. Blood.1995;86(2):813-818. 7. Weisdorf $\mathrm{D}$, et al. Treatment of moderate/severe acute graft-versus-host disease after allogeneic bone marrow transplantation: an analysis of clinical risk features and outcome. Blood.
1990;75(4):1024-1030.

8. Matte CC, et al. Donor APCs are required for maximal GVHD but not for GVL. Nat Med. 2004;10(9):987-992.

9. Shlomchik WD, et al. Prevention of graft versus host disease by inactivation of host antigen-presenting cells. Science. 1999;285(5426):412-415.

10. Sun Y, Tawara I, Toubai T, Reddy P. Pathophysiology of acute graft-versus-host disease: recent advances. Transl Res. 2007;150(4):197-214.

11. Glucksberg H, et al. Clinical manifestations of graft-versus-host disease in human recipients of marrow from HL-A-matched sibling donors. Transplantation. 1974;18(4):295-304.

12. Sullivan KM, et al. Chronic graft-versus-host 
disease and other late complications of bone marrow transplantation. Semin Hematol. 1991;28(3):250-259.

13. Filipovich $\mathrm{AH}$, et al. National Institutes of Health consensus development project on criteria for clinical trials in chronic graft-versus-host disease: I. Diagnosis and staging working group report. Biol Blood Marrow Transplant. 2005;11(12):945-956.

14. Shulman HM, et al. NIH consensus development project on criteria for clinical trials in chronic graft-versus-host disease: II. The 2014 Pathology Working Group Report. Biol Blood Marrow Transplant. 2015;21(4):589-603.

15. Hartwell MJ, et al. An early-biomarker algorithm predicts lethal graft-versus-host disease and survival. JCI Insight. 2017;2(3):e89798.

16. Lee SJ. Classification systems for chronic graftversus-host disease. Blood. 2017;129(1):30-37.

17. Storb R, et al. Methotrexate and cyclosporine compared with cyclosporine alone for prophylaxis of acute graft versus host disease after marrow transplantation for leukemia. N EnglJMed. 1986;314(12):729-735.

18. Socié $\mathrm{G}$, et al. Long-term survival and late deaths after allogeneic bone marrow transplantation. Late Effects Working Committee of the International Bone Marrow Transplant Registry. N EnglJ Med.1999;341(1):14-21.

19. Nichols WG, et al. Rising pp65 antigenemia during preemptive anticytomegalovirus therapy after allogeneic hematopoietic stem cell transplantation: risk factors, correlation with DNA load, and outcomes. Blood. 2001;97(4):867-874.

20. Marr KA, Carter RA, Boeckh M, Martin P, Corey L. Invasive aspergillosis in allogeneic stem cell transplant recipients: changes in epidemiology and risk factors. Blood. 2002;100(13):4358-4366.

21. Arai S, Margolis J, Zahurak M, Anders V, Vogelsang GB. Poor outcome in steroid-refractory graft-versus-host disease with antithymocyte globulin treatment. Biol Blood Marrow Transplant. 2002;8(3):155-160.

22. Andermann TM, et al. The microbiome and hematopoietic cell transplantation: past, present, and future. Biol Blood Marrow Transplant. 2018;24(7):1322-1340.

23. Shono Y, van den Brink MRM. Gut microbiota injury in allogeneic haematopoietic stem cell transplantation. Nat Rev Cancer. 2018;18(5):283-295.

24. Staffas A, Burgos da Silva M, van den Brink MR. The intestinal microbiota in allogeneic hematopoietic cell transplant and graft-versus-host disease. Blood. 2017;129(8):927-933.

25. Connell MS, Wilson R. The treatment of $\mathrm{x}$-irradiated germfree $\mathrm{CFW}$ and $\mathrm{C} 3 \mathrm{H}$ mice with isologous and homologous bone marrow. Life Sci (1962). 1965;4:721-729.

26. Jones JM, Wilson R, Bealmear PM. Mortality and gross pathology of secondary disease in germfree mouse radiation chimeras. Radiat Res. 1971;45(3):577-588.

27. van Bekkum DW, Roodenburg J, Heidt PJ, van der Waaij D. Mitigation of secondary disease of allogeneic mouse radiation chimeras by modification of the intestinal microflora. J Natl Cancer Inst. 1974;52(2):401-404.

28. Bealmear PM, Mirand EA, Holtermann OA. Modification of graft-vs-host disease following bone marrow transplantation in germfree mice. Prog Clin Biol Res. 1983;132C:409-421.

29. Wade AC, Luckert PH, Tazume S, Niedbalski JL, Pollard M. Characterization of xenogeneic mouse-to-rat bone marrow chimeras. I. Examination of hematologic and immunologic function. Transplantation. 1987;44(1):88-92.

30. Heidt PJ, Vossen JM. Experimental and clinical gnotobiotics: influence of the microflora on graftversus-host disease after allogeneic bone marrow transplantation. J Med.1992;23(3-4):161-173.

31. Bauer H, Horowitz RE, Levenson SM, Popper $\mathrm{H}$. The response of the lymphatic tissue to the microbial flora. Studies on germfree mice. Am J Pathol. 1963;42:471-483.

32. Hooper LV, Littman DR, Macpherson AJ. Interactions between the microbiota and the immune system. Science. 2012;336(6086):1268-1273.

33. Round JL, Mazmanian SK. The gut microbiota shapes intestinal immune responses during health and disease. Nat Rev Immunol. 2009;9(5):313-323.

34. Lampert IA, Moore RH, Huby R, Cohen J. Observations on the role of endotoxin in graft-versushost disease. Prog Clin Biol Res. 1988;272:351-359.

35. Vriesendorp HM, Heidt PJ, Zurcher C. Gastrointestinal decontamination of dogs treated with total body irradiation and bone marrow transplantation. Exp Hematol. 1981;9(9):904-916.

36. Jenq RR, et al. Regulation of intestinal inflammation by microbiota following allogeneic bone marrow transplantation. J Exp Med. 2012;209(5):903-911.

37. Simms-Waldrip TR, et al. Antibiotic-induced depletion of anti-inflammatory clostridia is associated with the development of graft-versus-host disease in pediatric stem cell transplantation patients. Biol Blood Marrow Transplant. 2017;23(5):820-829.

38. Buckner CD, et al. Protective environment for marrow transplant recipients: a prospective study. Ann Intern Med. 1978;89(6):893-901.

39. Navari RM, et al. Prophylaxis of infection in patients with aplastic anemia receiving allogeneic marrow transplants. Am JMed. 1984;76(4):564-572.

40. Storb R, et al. Graft-versus-host disease and survival in patients with aplastic anemia treated by marrow grafts from HLA-identical siblings. Beneficial effect of a protective environment. N Engl JMed.1983;308(6):302-307.

41. Petersen FB, et al. Laminar air flow isolation and decontamination: a prospective randomized study of the effects of prophylactic systemic antibiotics in bone marrow transplant patients. Infection. 1986;14(3):115-121.

42. Petersen FB, et al. Infectious complications in patients undergoing marrow transplantation: a prospective randomized study of the additional effect of decontamination and laminar air flow isolation among patients receiving prophylactic systemic antibiotics. Scand J Infect Dis. 1987;19(5):559-567.

43. Vossen JM, Heidt PJ, van den Berg H, Gerritsen EJ, Hermans J, Dooren LJ. Prevention of infection and graft-versus-host disease by suppression of intestinal microflora in children treated with allogeneic bone marrow transplantation. Eur JClin Microbiol Infect Dis. 1990;9(1):14-23.

44. Vossen JM, et al. Complete suppression of the gut microbiome prevents acute graft-versus-host disease following allogeneic bone marrow transplantation. PLoS One. 2014;9(9):e105706.

45. Beelen DW, et al. Evidence that sustained growth suppression of intestinal anaerobic bacteria reduces the risk of acute graft-versus-host disease after sibling marrow transplantation. Blood. 1992;80(10):2668-2676.

46. Beelen DW, Elmaagacli A, Müller KD, Hirche $\mathrm{H}$, Schaefer UW. Influence of intestinal bacterial decontamination using metronidazole and ciprofloxacin or ciprofloxacin alone on the development of acute graft-versus-host disease after marrow transplantation in patients with hematologic malignancies: final results and long-term follow-up of an open-label prospective randomized trial. Blood. 1999;93(10):3267-3275.

47. Guthery SL, Heubi JE, Filipovich A. Enteral metronidazole for the prevention of graft versus host disease in pediatric marrow transplant recipients: results of a pilot study. Bone Marrow Transplant. 2004;33(12):1235-1239.

48. Taur Y, et al. The effects of intestinal tract bacterial diversity on mortality following allogeneic hematopoietic stem cell transplantation. Blood. 2014;124(7):1174-1182.

49. Jenq RR, et al. Intestinal blautia is associated with reduced death from graft-versus-host disease. Biol Blood Marrow Transplant. 2015;21(8):1373-1383.

50. Gerbitz A, et al. Probiotic effects on experimental graft-versus-host disease: let them eat yogurt. Blood. 2004;103(11):4365-4367.

51. Gorshein E, et al. Lactobacillus rhamnosus GG probiotic enteric regimen does not appreciably alter the gut microbiome or provide protection against GVHD after allogeneic hematopoietic stem cell transplantation. Clin Transplant. 2017;31(5):e12947.

52. Holler E, et al. Metagenomic analysis of the stool microbiome in patients receiving allogeneic stem cell transplantation: loss of diversity is associated with use of systemic antibiotics and more pronounced in gastrointestinal graft-versus-host disease. Biol Blood Marrow Transplant. 2014;20(5):640-645.

53. Weber $\mathrm{D}$, et al. Low urinary indoxyl sulfate levels early after transplantation reflect a disrupted microbiome and are associated with poor outcome. Blood. 2015;126(14):1723-1728.

54. Biagi E, et al. Gut microbiota trajectory in pediatric patients undergoing hematopoietic SCT. Bone Marrow Transplant. 2015;50(7):992-998.

55. Doki N, et al. Clinical impact of pre-transplant gut microbial diversity on outcomes of allogeneic hematopoietic stem cell transplantation. Ann Hematol. 2017;96(9):1517-1523.

56. Golob JL, et al. Stool microbiota at neutrophil recovery is predictive for severe acute graft vs host disease after hematopoietic cell transplantation. Clin Infect Dis. 2017;65(12):1984-1991.

57. Liu C, et al. Associations between acute gastrointestinal GvHD and the baseline gut microbiota of allogeneic hematopoietic stem cell transplant recipients and donors. Bone Marrow Transplant. 2017;52(12):1643-1650.

58. Kokai-Kun JF. SYN-004 (ribaxamase) prevents Clostridium difficile infection and antimicrobial resistance. Presented at: Anaerobe Conference; July 12, 2018; Las Vegas, Nevada, USA. https:// 
www.syntheticbiologics.com/news-media/scientific-publications. Accessed January 2, 2019.

59. Kokai-Kun JF, et al. The oral $\beta$-lactamase SYN-OO 4 (ribaxamase) degrades ceftriaxone excreted into the intestine in phase $2 \mathrm{a}$ clinical studies. Antimicrob Agents Chemother. 2017;61(3):e02197-e02116.

60. Cooke KR, et al. LPS antagonism reduces graft-versus-host disease and preserves graftversus-leukemia activity after experimental bone marrow transplantation. J Clin Invest. 2001;107(12):1581-1589.

61. Schwab L, et al. Neutrophil granulocytes recruited upon translocation of intestinal bacteria enhance graft-versus-host disease via tissue damage. Nat Med. 2014;20(6):648-654.

62. Smith PM, et al. The microbial metabolites, short-chain fatty acids, regulate colonic Treg cell homeostasis. Science. 2013;341(6145):569-573.

63. Vinolo MA, Rodrigues HG, Nachbar RT, Curi R. Regulation of inflammation by short chain fatty acids. Nutrients. 2011;3(10):858-876.

64. Ma X, Fan PX, Li LS, Qiao SY, Zhang GL, Li DF. Butyrate promotes the recovering of intestinal wound healing through its positive effect on the tight junctions. JAnim Sci. 2012;90(suppl 4):266-268.

65. Gilbert KM, Boger S, Fifer EK. Butyric acid derivative induces allospecific T cell anergy and prevents graft-versus-host disease. Immunopharmacol Immunotoxicol. 2003;25(1):13-27.

66. Mathewson ND, et al. Gut microbiome-derived metabolites modulate intestinal epithelial cell damage and mitigate graft-versus-host disease. Nat Immunol. 2016;17(5):505-513.

67. Gilbert KM, Boger SR, Price P, Fifer EK. T cell tolerance induced by histone deacetylase inhibitor is mediated by P21cip1. Immunopharmacol Immunotoxicol. 2005;27(4):545-564.

68. Soderberg LS, Boger S, Fifer EK, Gilbert KM. Macrophage production of inflammatory mediators is potently inhibited by a butyric acid derivative demonstrated to inactivate antigenstimulated T cells. Int Immunopharmacol. 2004;4(9):1231-1239.

69. Choi SW, et al. Histone deacetylase inhibition regulates inflammation and enhances Tregs after allogeneic hematopoietic cell transplantation in humans. Blood. 2015;125(5):815-819.

70. Furusawa Y, et al. Commensal microbe-derived butyrate induces the differentiation of colonic regulatory T cells. Nature. 2013;504(7480):446-450.

71. Eriguchi Y, et al. Decreased secretion of Paneth cell $\alpha$-defensins in graft-versus-host disease. Transpl Infect Dis. 2015;17(5):702-706.

72. Eriguchi Y, et al. Graft-versus-host disease disrupts intestinal microbial ecology by inhibiting Paneth cell production of $\alpha$-defensins. Blood . 2012;120(1):223-231.

73. Hayase E, et al. R-Spondin1 expands Paneth cells and prevents dysbiosis induced by graft-versushost disease. JExp Med. 2017;214(12):3507-3518.

74. Levine JE, et al. Low Paneth cell numbers at onset of gastrointestinal graft-versus-host disease identify patients at high risk for nonrelapse mortality. Blood. 2013;122(8):1505-1509.

75. Ara T, et al. Degree of intestinal goblet-cell loss is associated with severity of GvHD and transplant outcome, and goblet cell growth factor IL-25 mitigates GvHD. Biol Blood Marrow Transplant.
2018;24(3):S169-S170.

76. Heidegger S, van den Brink MR, Haas T, Poeck H. The role of pattern-recognition receptors in graftversus-host disease and graft-versus-leukemia after allogeneic stem cell transplantation. Front Immunol. 2014;5:337.

77. Heimesaat MM, et al. MyD88/TLR9 mediated immunopathology and gut microbiota dynamics in a novel murine model of intestinal graft-versus-host disease. Gut. 2010;59(8):1079-1087.

78. Moore RH, Lampert IA, Chia Y, Aber VR, Cohen $\mathrm{J}$. Influence of endotoxin on graft-versus-host disease after bone marrow transplantation across major histocompatibility barriers in mice. Transplantation. 1987;43(5):731-736.

79. Rayes A, Morrow AL, Payton LR, Lake KE, Lane A, Davies SM. A genetic modifier of the gut microbiome influences the risk of graft-versushost disease and bacteremia after hematopoietic stem cell transplantation. Biol Blood Marrow Transplant. 2016;22(3):418-422.

80. Taur Y, Jenq RR, Ubeda C, van den Brink M, Pamer EG. Role of intestinal microbiota in transplantation outcomes. Best Pract Res Clin Haematol. 2015;28(2-3):155-161.

81. Tawara I, et al. Influence of donor microbiota on the severity of experimental graft-versushost-disease. Biol Blood Marrow Transplant. 2013;19(1):164-168.

82. Teshima T, Reddy P, Zeiser R. Acute graft-versushost disease: novel biological insights. Biol Blood Marrow Transplant. 2016;22(1):11-16.

83. Aguilar-Guisado M, et al. Optimisation of empirical antimicrobial therapy in patients with haematological malignancies and febrile neutropenia (How Long study): an open-label, randomised, controlled phase 4 trial. Lancet Haematol. 2017;4(12):e573-e583.

84. Weber D, et al. Microbiota disruption induced by early use of broad-spectrum antibiotics is an independent risk factor of outcome after allogeneic stem cell transplantation. Biol Blood Marrow Transplant. 2017;23(5):845-852.

85. DeFilipp Z, et al. Third-party fecal microbiota transplantation following allo-HCT reconstitutes microbiome diversity. Blood Adv. 2018;2(7):745-753.

86. Routy B, et al. The influence of gut-decontamination prophylactic antibiotics on acute graftversus-host disease and survival following allogeneic hematopoietic stem cell transplantation. Oncoimmunology. 2017;6(1):e1258506.

87. Eloe-Fadrosh EA, et al. Functional dynamics of the gut microbiome in elderly people during probiotic consumption. MBio. 2015;6(2):e00231-15.

88. Chang JY, et al. Decreased diversity of the fecal microbiome in recurrent Clostridium difficile-associated diarrhea. JInfect Dis. 2008;197(3):435-438.

89. Fischer JC, et al. RIG-I/MAVS and STING signaling promote gut integrity during irradiation- and immune-mediated tissue injury. Sci Transl Med. 2017;9(386):eaag2513.

90. Byndloss MX, et al. Microbiota-activated PPAR- $\gamma$ signaling inhibits dysbiotic Enterobacteriaceae expansion. Science. 2017;357(6351):570-575.

91. Arpaia N, et al. Metabolites produced by commensal bacteria promote peripheral regulatory T-cell generation. Nature. 2013;504(7480):451-455.

92. Atarashi K, et al. Treg induction by a ratio- nally selected mixture of Clostridia strains from the human microbiota. Nature. 2013;500(7461):232-236.

93. Shono Y, et al. Increased GVHD-related mortality with broad-spectrum antibiotic use after allogeneic hematopoietic stem cell transplantation in human patients and mice. Sci Transl Med. 2016;8(339):339ra71.

94. Hülsdünker J, et al. Neutrophils provide cellular communication between ileum and mesenteric lymph nodes at graft-versus-host disease onset. Blood. 2018;131(16):1858-1869.

95. Varelias A, et al. Acute graft-versus-host disease is regulated by an IL-17-sensitive microbiome. Blood. 2017;129(15):2172-2185.

96. Varelias A, et al. Recipient mucosal-associated invariant $\mathrm{T}$ cells control GVHD within the colon. JClin Invest. 2018;128(5):1919-1936.

97. Bhattacharyya A, et al. Graft-derived reconstitution of mucosal-associated invariant T cells after allogeneic hematopoietic cell transplantation. Biol Blood Marrow Transplant. 2018;24(2):242-251.

98. Zelante T, et al. Tryptophan catabolites from microbiota engage aryl hydrocarbon receptor and balance mucosal reactivity via interleukin-22. Immunity. 2013;39(2):372-385.

99. Lindemans CA, et al. Interleukin-22 promotes intestinal-stem-cell-mediated epithelial regeneration. Nature. 2015;528(7583):560-564.

100.Hanash AM, et al. Interleukin-22 protects intestinal stem cells from immune-mediated tissue damage and regulates sensitivity to graft versus host disease. Immunity. 2012;37(2):339-350.

101.Zenewicz LA, et al. IL-22 deficiency alters colonic microbiota to be transmissible and colitogenic. J Immunol. 2013;190(10):5306-5312.

102. Kakihana K, et al. Fecal microbiota transplantation for patients with steroid-resistant acute graft-versus-host disease of the gut. Blood 2016;128(16):2083-2088.

103. Spindelboeck W, et al. Repeated fecal microbiota transplantations attenuate diarrhea and lead to sustained changes in the fecal microbiota in acute, refractory gastrointestinal graft-versus-hostdisease. Haematologica. 2017;102(5):e210-e213.

104. Taur Y, et al. Reconstitution of the gut microbiota of antibiotic-treated patients by autologous fecal microbiota transplant. Sci Transl Med. 2018;10(460):eaap9489.

105. Ladas EJ, et al. The safety and feasibility of probiotics in children and adolescents undergoing hematopoietic cell transplantation. Bone Marrow Transplant. 2016;51(2):262-266.

106. Mattsson J, Westin S, Edlund S, Remberger M. Poor oral nutrition after allogeneic stem cell transplantation correlates significantly with severe graft-versus-host disease. Bone Marrow Transplant. 2006;38(9):629-633.

107. Weber D, et al. Rifaximin preserves intestinal microbiota balance in patients undergoing allogeneic stem cell transplantation. Bone Marrow Transplant. 2016;51(8):1087-1092.

108. Pomerantz RJ. Seres Therapeutics, Inc. Presented at: 38th Annual Cowen \& Co. Health Care Conference; March 14, 2018; Boston, Massachusetts, USA. http://ir.serestherapeutics.com/phoenix. zhtml? $\mathrm{c}=254006 \& \mathrm{p}=$ irol-EventDetails\&EventId = 5269478. Accessed January 2, 2019. 